\title{
Construyendo muestras artificiales con Mathematica
}

\author{
Sonia I Mariño \\ simarinio@yahoo.com \\ Universidad Nacional del Nordeste. \\ Argentina
}

\author{
María Victoria López \\ mvlopez@exa.unne.edu.ar \\ Universidad Nacional del Nordeste. \\ Argentina
}

\section{Resumen.}

Se describe un módulo del Entorno Virtual de Enseñanza Aprendizaje (EVEA) de la asignatura Modelos y Simulación de la carrera de Licenciatura en Sistemas de Información, en la Facultad de Ciencias Exactas y Naturales y Agrimensura de la Universidad Nacional del Nordeste (FACENA-UNNE) en Corrientes, Argentina. Su propósito es la formación de los estudiantes en la aplicación de la técnica de simulación para la resolución de problemas basados en casos reales. Este artículo se compone de cinco secciones. En la primera sección se caracteriza la asignatura objeto de estudio y se mencionan antecedentes sobre la aplicación de las Tecnologías de la Información y Comunicación (TIC) como apoyo al proceso de enseñanza-aprendizaje o a los procesos de modelización y simulación de problemas. En la segunda sección se describe la metodología adoptada en el desarrollo de la experiencia que se describe en este paper. En la tercera sección se detalla el perfil de los estudiantes que optan por esta asignatura. En la cuarta sección se sintetizan las funcionalidades del módulo de muestras artificiales generado con Mathematica y la descripción de su implementación en el aula. En la última sección, se comentan algunas conclusiones y futuros trabajos.

Palabras claves: Matemática aplicada, Modelos y Simulación, Muestras artificiales, Educación superior, EVEA, Enseñanza de la matemática asistida por computadora, Software Mathematica.

\begin{abstract}
.
We describe a module of the Virtual Learning Environment (VLE) of the course Modelos y Simulación of the Licenciatura en Sistemas de Información career, from FACENA-UNNE in Corrientes, Argentina. Its purpose is to train students in the application of simulation techniques for solving problems based on real cases. The paper consists of five sections. The first section characterizes the subject under study and are mentioned background on the application of Information Technologies and Communication Technologies (ICTs) to support the teaching-learning process or modeling and simulation problems. The second section describes the methodology adopted in the development of the experience described in this paper. The third section details the profile of students who opt for this course. The fourth section summarizes the functionality of the module of artificial samples generated with Mathematica and the description of its implementation in the classroom. In the last section, we discuss some conclusions and future work.
\end{abstract}

KeyWords: Applied mathematics, Modeling and simulation, Artificial samples, Higher Education, VLE, Computer-assisted mathematics teaching, Mathematica Software. 


\subsection{Introducción.}

"Modelos y Simulación" es una asignatura optativa (Optativa II) del plan de estudios de la carrera de Licenciatura en Sistemas de Información (LSI) de la Facultad de Ciencias Exactas y Naturales y Agrimensura de la Universidad Nacional del Nordeste (FACENA-UNNE) en Argentina. El objetivo general de la asignatura es proporcionar una formación sólida en el manejo de los conceptos y técnicas utilizadas en la simulación de sistemas mediante el procesamiento digital de modelos matemáticos. Se enfatizan la búsqueda y la solución de problemas científicos y profesionales aplicando técnicas específicas.

Los contenidos abordados pertenecen al campo de la Matemática Aplicada. Puede caraterizarse como una asignatura de formación complementaria siguiendo a [13]. Es decir, brinda los conocimientos, habilidades y valores que otorgan al estudiante una visión más amplia de su profesión y del mundo.

En [27] se comenta que "la técnica de modelado y simulación de sistemas permite crear ambientes virtuales que imitan el comportamiento de prácticamente cualquier tipo de sistema, a efectos de evaluar su desempeño minimizando los costos de la toma de decisiones".

La asignatura se compone de cuatro grandes ejes temáticos o disciplinares. El primero comprende las unidades donde se introducen los temas de sistemas, modelos, simulación y metodología de un estudio de simulación. El segundo eje aborda la generación de series de números pseudoaleatorios. El tercer eje temático trata la construcción de muestras artificiales representativas de distintas distribuciones de probabilidad, discretas y continuas. El cuarto eje integra los conceptos teóricos y prácticos vistos anteriormente, plasmados en la construcción de modelos de simulación. Este trabajo se aborda/focaliza en los ejes temáticos dos y tres mediante la generación de muestras artificiales con Mathematica

Esta asignatura proporciona a los alumnos conocimientos sobre el desarrollo de modelos de tipo matemático, los cuales son utilizados para simular una amplia gama de sistemas reales. Estos conocimientos deben necesariamente ser complementados con los adquiridos en otras asignaturas (lenguajes de programación, paradigmas de desarrollo, técnicas de análisis de sistemas, cálculo de probabilidades y estadística) para resolver los Trabajos Prácticos propuestos. Se requiere un razonamiento inteligente por parte de los alumnos para seleccionar aquellos lenguajes y modelos que mejor se adapten a la resolución del problema que se les presenta. Se pretende generar un trabajo original y creativo que propicie en los alumnos la utilización de las distintas herramientas tecnológicas y los conocimientos con los que dispone, a partir de un proceso de aprendizaje que se inició al comenzar la Carrera.

Los alumnos inscriptos pueden regularizarla o promocionarla. Las condiciones de regularización y promoción son similares, consistiendo en: asistencia al 75\% de las clases teóricas-practicas y prácticas de laboratorio, aprobación de dos parciales o sus correspondientes recuperatorios, presentación de un trabajo integrador y su exposición. La diferencia reside en que regularizan la asignatura quienes no superan una calificación igual o superior al 7 en cada una de las evaluaciones.

Como se expresó en trabajos previos [1], [2], [17], [34], [35], [36] y según lo relevado en diversas publicaciones [6], [7], [9], [10], [37], las Tecnologías de la Información y Comunicación (TIC) se pueden emplear en dos sentidos: como apoyo al proceso de enseñanza-aprendizaje o para apoyar a los procesos de modelización y simulación de problemas basados en casos reales. 
En el primer sentido pueden mediatizarse mediante los Entornos Virtuales de Enseñanza Aprendizaje (EVEA) o como herramientas para apoyar el aprendizaje mixto o $b$-learning. En el segundo sentido como herramientas o recursos para la construcción de modelos y/o simuladores.

La modalidad de enseñanza y aprendizaje denominada b-learning, se emplea para referenciar la combinación de elementos de la enseñanza presencial tradicional y la enseñanza a distancia, empleando las TIC basadas en la web. Como lo expresa [14] "combina lo mejor de la instrucción presencial con funcionalidades de e-learning, para potenciar las fortalezas y disminuir las debilidades de ambas modalidades". Por su parte, [11] expresa otras denominaciones del término como ser: "enseñanza bi-modal, mixta, hibrida, semi-presencial, o web-asistida.

La modalidad b-learning es una alternativa viable en la actual educación superior [4], [29]. Es posible emplearla como "complemento de las clases y soporte documental" [4].

En la asignatura Modelos y Simulación, desde el año 2005, se aplica la modalidad de aprendizaje combinado o blended learning caracterizada en trabajos descriptos por [16], [20], [21].

Este trabajo se enmarca en las acciones de docencia, extensión e investigación impulsadas desde la cátedra Modelos y Simulación [23], [25], especialmente las vinculadas con la aplicación de las TIC plasmadas en innovaciones pedagógicas y la elaboración de materiales didácticos en diversos formatos. Se describe un módulo a incorporarse al EVEA de la asignatura Modelos y Simulación, el cual incluye

a) Un paquete en Mathematica, con procedimientos para generar muestras artificiales de variables aleatorias discretas y continuas empleando métodos especiales.

b) Simuladores del funcionamiento del mencionado paquete.

c) Autoevaluaciones que permite a los estudiantes afianzar y validar sus conocimientos

\subsection{Metodología}

En esta sección se expone la metodología elaborada ad-hoc que fuera utilizada en el diseño y desarrollo de diversos paquetes de software de enseñanza-aprendizaje aplicables en el ámbito de la asignatura [24], y que se adopta en la construcción de los recursos abordados en este trabajo.

a) Estudio de factibilidad. Consiste en una estimación de recursos necesarios para apoyar el aprendizaje significativo de los temas y conceptos tratados. Permite establecer claramente los límites del entorno virtual y su integración con otros entornos similares aplicables en la asignatura. Primeramente, como paso fundamental y previo a la etapa de selección de la herramienta, se observaron las necesidades del EVEA y qué aplicabilidad tendría, para luego acotar más el espectro que definiría los posibles lenguajes o herramientas que serían utilizados a tal efecto. Las necesidades requeridas en la asignatura se vinculan con el diseño y desarrollo de complementos para apoyar el proceso de aprendizaje de la asignatura Modelos y Simulación.

b) Definición de los destinatarios. Al diseñar un software, un interrogante muy importante que se debe plantear es: ¿Quiénes utilizarán el software? Los destinatarios de este software interactivo son los alumnos de la asignatura Modelos y Simulación de la carrera de Licenciatura en Sistemas de Información de la FACENA - UNNE. Realizada la delimitación geográfica, se puede decir que el software podrá ser utilizado en los laboratorios de la institución, convirtiéndose de esta manera en una herramienta de apoyo fuera del horario del cursado de la asignatura. 
c) Identificación de los requerimientos. En esta etapa de la construcción de los materiales instruccionales interactivos se establece de manera clara y precisa el conjunto de requisitos que debe satisfacer el software. Desde el punto de vista del rendimiento, éste debe generar series de números pseudoaleatorios y muestras artificiales en lapsos muy breves de tiempo. Para brindar una visión más clarificadora de los requerimientos del sistema se recurre a técnicas de modelado UML (Unified Modeling Language).

d) Definición de la arquitectura general o infraestructura. Desde el punto de vista de la arquitectura o infraestructura sobre la cual se ejecuta el software, los procedimientos requieren del producto Mathematica.

e) Selección del medio de distribución. Se deben tener en cuenta las características del desarrollo, respecto a la forma de ejecución y tamaño, a la hora de decidir el medio en el cual será distribuido.

f) Análisis del entorno virtual. Luego de realizar el estudio de los aspectos fundamentales del software educativo, se logra una visión más clara del entorno que éste debe presentar.

g) Diseño del entorno virtual. Se contemplan características como: i) Interactividad, ii) Integración de contenidos en múltiples formatos, iii) Definición del objetivo de implementación. En el diseño de las interfaces se deben considerar la navegabilidad, accesibilidad y comunicación, y su especificación en el desarrollo de entornos virtuales de enseñanza-aprendizaje.

h) Selección y evaluación de herramientas. El análisis de las herramientas de software permite obtener una visión más concreta de las funcionalidades y características más importantes de las mismas, e identificar cuáles de ellas posibilitan dar un enfoque más sencillo y práctico de los problemas de simulación abordados. Para la construcción de los simuladores se evaluaron una diversidad de herramientas de programación como Flash, MatLab, Visual Basic, Java, Octave, Mathematica, entre otros, seleccionándose finalmente el último. Se ha optado por este lenguaje de programación, porque provee un entorno de desarrollo con herramientas y facilidades que ayudan a la productividad al momento de programar las funciones y graficar resultados. El desarrollo que se describe se integra al EVEA distribuible desde diversos medios, ya sea vía web o en dispositivos digitales como cd-rom o dvd-rom.

i) Selección y preparación de contenidos. Los contenidos incorporados al entorno virtual tienen como finalidad facilitar y/o complementar el desarrollo de las clases presenciales de la asignatura [23]. En este caso en particular, se incorporaron el paquete en Mathematica, los simuladores del funcionamiento y las autoevaluaciones vinculadas con los métodos especiales para generar muestras artificiales de variables aleatorias discretas y continuas. Los algoritmos que implementan los métodos mencionados, que fueron codificados en Mathematica, se basaron en textos disciplinares [5], [15], [22], [26], [30], [31], [32].

j) Desarrollo del entorno virłual. Este trabajo requirió la incorporación de una opción dentro del EVEA desde la cual sea accesible el caso experimental abordado.

k) Incorporación de contenidos. Se incorpora este caso de estudio al entorno virtual de la cátedra, con miras a contribuir como contenido didáctico.

I) Validaciones. Finalizado el desarrollo, se verificó el correcto funcionamiento del sistema y el acceso a los contenidos. Con respecto al funcionamiento se comprueba: i) Mapa de navegación. Estructuración que permite acceder a los contenidos, actividades, niveles y prestaciones en general. ii) Sistema de navegación. Entorno transparente que permite al usuario tener el control. iii) Velocidad entre el usuario y el programa (animaciones, lectura de datos, etc.). iv) Ejecución de los programas incluidos para actuar como simuladores de lo problemas abordados. 


\subsection{Perfil del estudiante que opta por la asignatura Modelos y simulación}

Desde el año 2005 al 2009 se han relevado datos a fin de caracterizar el perfil del alumno y que se comentaron en trabajos previos [17], [18],[21].

Se observa una muy buena relación entre el número de alumnos inscriptos y el número de alumnos promovidos sin examen final (Tabla 1). Esto puede deberse a las siguientes causas:

a) La franja de edad de los alumnos de la asignatura es entre 20 y 40 años aproximadamente. Como se trata de alumnos del tercer año de la Carrera (segundo ciclo), y que en su mayoría poseen un Título de Nivel Intermedio (80 \% aprox), un alto porcentaje de los mismos posee un empleo (70 \% aprox), lo que implica que no dedican el cien por ciento de su tiempo al estudio. Se observa que los alumnos que trabajan poseen mayor habilidad y destreza en el manejo de lenguajes de programación que los que no lo hacen, aunque a veces dedican menos tiempo al estudio de los conceptos teóricos de la asignatura, y en este caso se requiere el cumplimiento de ambos aspectos. Los alumnos que trabajan comúnmente no pueden concurrir a clases presenciales, por tanto la implementación de alternativas complementarias a los materiales impresos, tales como un entorno virtual de la asignatura, empleando como soportes la Web y el CD-ROM, constituyen un buen recurso atendiendo a la realidad de los cursantes.

b) El número reducido de alumnos permite trabajar personalizadamente e implementar procedimientos alternativos de evaluación de los aprendizajes, tal como la técnica del portfolio [12], referida a una compilación de trabajos del alumno, recogidos a lo largo del tiempo, que aportan evidencias respecto de sus conocimientos, habilidades y de su disposición para actuar de determinadas maneras. En la última etapa del dictado de la asignatura, los alumnos deberán realizar los trabajos prácticos individuales preparados por los docentes denominados Trabajos de seminario. Estos integran todos los contenidos teórico-prácticos abordados, mediante la modelización y construcción de simulaciones representativas de casos reales. De este modo, los alumnos podrán integrar o reutilizar los programas desarrollados en las clases de laboratorio en el producto final del portfolio, el cual consistiría en un software simulador acompañado de un informe descriptivo del trabajo realizado.

c) Desde la asignatura, se realizan numerosas actividades de tutorías y seguimiento continuo en modalidad presencial o virtual a través del correo electrónico.

Tabla 1.1 Alumnos inscriptos, regulares y promocionales en las cohortes 2005-2009 de la asignatura "Modelos y Simulación"

\begin{tabular}{|l|l|l|l|}
\hline Cohorte & Al & AR & AP \\
\hline 2005 & 24 & 1 & 16 \\
\hline 2007 & 37 & 2 & 18 \\
\hline 2008 & 58 & 3 & 22 \\
\hline 2009 & 34 & 2 & 19 \\
\hline
\end{tabular}

AI: alumnos inscriptos en la asignatura.

AR: alumnos que regularizaron la asignatura.

AP: alumnos que promocionaron la asignatura.

En [3] se mencionan cuatro criterios que permiten decidir qué recursos utilizar en la modalidad de b-learning: i) Condiciones requeridas para la formación demandada: urgencia, objetivos, necesidad de resultados ob- 
servables; ii) Recursos disponibles; iii) Características de los usuarios estudiantiles; iv) Características del contenido educativo.

Lo expuesto indica la necesidad de evaluar continuamente el material producido así como el alumnado, ya que son condicionantes para lograr resultados exitosos en el proceso de enseñanza - aprendizaje.

A continuación se resume el relevamiento de datos concretado en el año 2009. Cabe aclarar la buena predisposición de los estudiantes al devolver los cuestionarios con las respuestas. Los resultados del relevamiento efectuado se exponen en las Figuras 1.1 a 1.6.

Se aplicó una encuesta a un total de 17 estudiantes que optaron por esta asignatura en el año 2009. Se detectó que la edad mínima fue de 22 y la máxima de 39, estimándose una edad promedio igual a 27 años, variando la fecha de ingreso a la educación superior entre los años 1997 y 2006.

Del total de alumnos, el 47,05\% corresponde al sexo femenino, mientras que un 52,95 \% al sexo masculino (Figura 1.1).

Al ser cuestionados en referencia al número de asignaturas que adeudan para la obtención del título de Licenciado en Sistemas de Información (LSI), se detectó que un 82,35 \% de los alumnos adeuda más de seis asignaturas, un 11,76\% adeuda entre tres y seis asignaturas, y un 5,88 \% menos de cuatro asignaturas (Figura $1.2)$.

Asimismo, se determinó que el 52,94\% de los estudiantes cuenta con el título intermedio de Programador Universitario de Aplicaciones (PUA), mientras que el 41,18\% todavía no ha obtenido el mencionado título, y un 5,88\% no respondió (Figura 1.3).

Atendiendo a que Modelos y Simulación es la segunda de cuatro asignaturas optativas, se consideró interesante determinar qué asignatura optativa eligieron los alumnos en el primer cuatrimestre del tercer año de estudios. El procesamiento permitió determinar que un 5,88 \% de los alumnos optó por Arquitectura de Computadores (AC), un 41,18 \% por Conceptos Teóricos Básicos (CTB), un 17,65 \% prefirió Gestión de Centros de Cómputos (GCC), un 11,76 \% Investigación Operativa (IO), mientras que un 17,65\% no respondió (NC). Este análisis permite afirmar que es escasa la vinculación entre asignaturas previas optativas, dificultándose la generación de trayectos o líneas de estudio dentro de una misma área temática. En la Figura 1.4 se representa en porcentajes las elecciones de los alumnos, las iniciales de las leyendas corresponden a las asignaturas que componen la oferta de la Optativa II: AC: Arquitectura de Computadoras, GCC: Gestión de Centro de Cómputos, CTB: Conceptos Teóricos Básico, NC: No Contesta.

$\mathrm{Al}$ encuestar respecto a los conocimientos previos, el 47,06 \% de los alumnos considera que son suficientes o medianamente suficientes, mientras que el 5,88 \% de los mismos los considera insuficientes. Asimismo, el $88,24 \%$ de los estudiantes opina que vinculó con conocimientos previos, mientras que el 5,88\% respondió que escasamente o que no logró la vinculación esperada (Figura 1.5). Se determinó que el 88,24 \% de los alumnos logró la vinculación interna de los contenidos abordados en la teoría con los con los ejemplos y ejercicios prácticos planteados mientras que un $11,76 \%$ alcanzó en términos medios.

Por otra parte, con miras a lograr la formación de profesionales que se inserten en el mercado laboral, resulta de especial interés que los estudiantes vinculen los contenidos abordados con ejemplos o situaciones de la vida cotidiana, lográndose esto en un $94,12 \%$ de los casos y en términos medios en un 5,88 \% de los casos. Mayoritariamente, mencionaron la vinculación con temas de simulación de colas, inventarios y juegos de azar. 
La asignatura se caracteriza por la disponibilidad de diversos recursos orientados a afianzar el proceso de enseñanza-aprendizaje. Al evaluar los trabajos prácticos y ensayos de laboratorios propuestos (en una escala con las siguientes categorías: muy bueno, bueno, regular, malo, muy malo), un 94,12\% de los alumnos respondió que éstos permitieron entender los temas abordados, mientras que un 5,88 \% respondió que posibilitaron la comprensión de los contenidos en términos medios. Se aplicó la misma escala en la evaluación del material disponible en el EVEA (cd) e impreso. Un 64,71\% de los estudiantes opinaron que el material didáctico compilado en el CD es muy bueno, un 29,41\% consideró que es bueno y un 5,88 \% lo calificó como regular (Figura 1.6). Con respecto del material impreso, el 52,94 \% de los alumnos respondió que es muy bueno, y el 41,18\% que es bueno, mientras que un $5.88 \%$ lo consideró regular.

En referencia a los mecanismos de evaluación empleados en la asignatura, el 100\% de los alumnos consideró que los mismos son adecuados, incluso la propuesta innovadora aplicada consistente en un parcial domiciliario en el cual debían plantear una situación problemática y resolverla mediante la generación de una muestra artificial. El parcial domiciliario consistió en la elaboración de un enunciado de un problema abstraído de una situación del mundo real para su modelización y simulación aplicando las técnicas y herramientas abordadas en la asignatura. Su implementación se fundamenta en que el equipo docente considera que es así como se logra el aprendizaje y afianzamiento de los conceptos tratados en el curso. Los alumnos dispusieron de una semana para su desarrollo fuera del aula. Cabe aclarar que las consignas de elaboración y la puntuación asignada son explicitadas a los alumnos previamente.

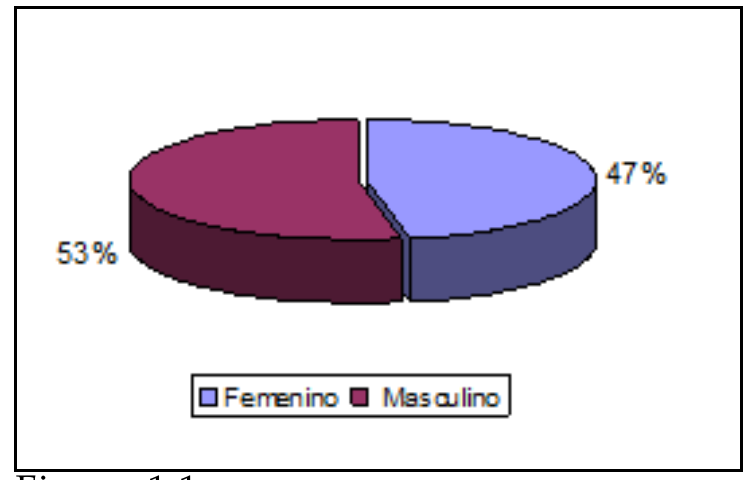

Figura 1.1: Género de los alumnos

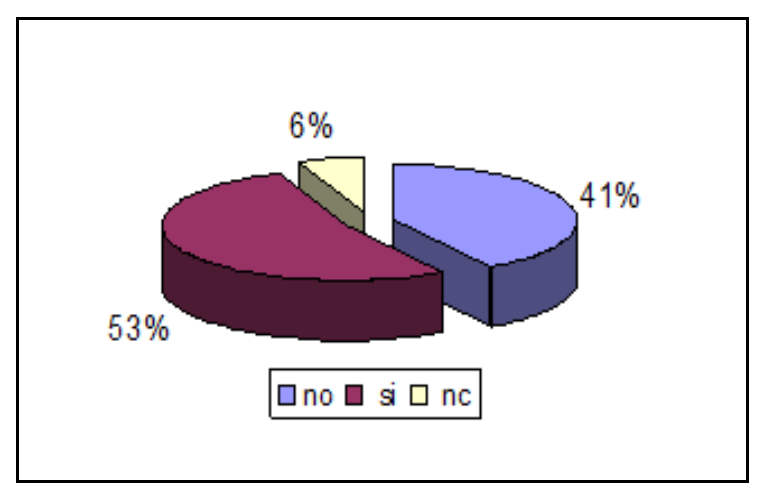

Figura 1.3: Alumnos que poseen el título intermedio de PUA

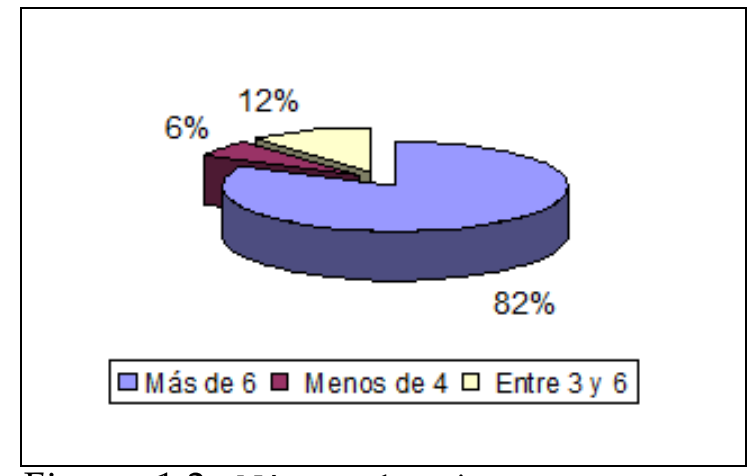

Figura 1.2: Número de asignaturas que adeudan para la obtención del título de LSI

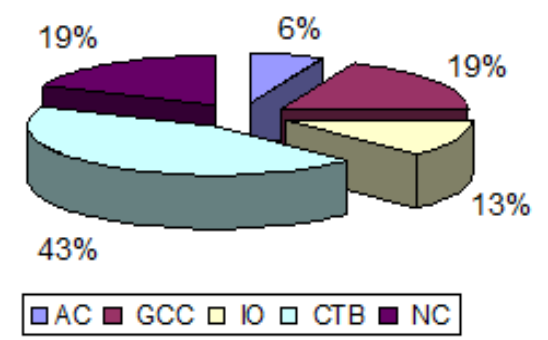

Figura 1.4: As ignatura elegida por los a lumnos como Optativa I 


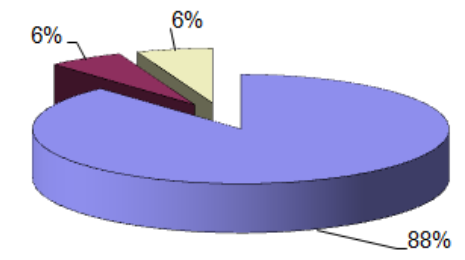

$\square$ Si $\square$ Escasamente $\square$ En términos medios

Figura 1.5: Vinculación de los contenidos de la as ignatura con conocimientos previos

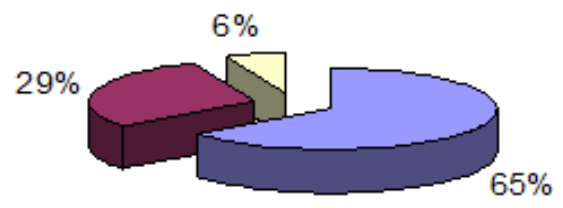

$\square$ Muy Bueno $\square$ Bueno $\square$ Regular

Figura 1.6: Opinión con respecto al CD de la as ignatura

\subsection{Resultados o Desarrollos}

Los docentes de la asignatura Modelos y Simulación diseñaron y desarrollaron un EVEA, utilizado como recurso complementario en el dictado de las clases. Este material se encuentra disponible para la consulta de los alumnos en el Laboratorio de Informática y en la Biblioteca de la Facultad en formatos digital e impreso, y dispone de las siguientes categorías de información:

a) Presentación de la asignatura: Ubicación de la asignatura en el plan de estudios de la carrera de Licenciatura en Sistemas de Información, modalidad y objetivos generales de dictado.

b) Programa analítico.

c) Planificación de actividades.

d) Composición del equipo docente.

e) Bibliografía y sitios web: Se proporciona a los alumnos bibliografía y direcciones de Internet que contengan material didáctico y ejercicios sobre los temas del programa de la asignatura.

f) Contenidos teóricos: Cada tema del programa se encuentra desarrollado con adecuado grado de detalle y profundización.

g) Aplicaciones prácticas: Se presentan temas agrupados en los tres ejes principales de la asignatura: números aleatorios, muestras artificiales y modelos de simulación. Para cada tema se puede acceder a: una breve descripción del mismo, ejercicios prácticos de ejemplo resueltos (diagramas de flujo), descripción del software que implementa el tema, y un enlace para efectuar la descarga del software.

h) Series de Trabajos Prácticos: Desde este enlace se puede acceder a las Guías de Trabajos Prácticos y Guías de ensayos de Laboratorio, a utilizar en las clases teórico-prácticas y en las clases de laboratorio respectivamente.

i) Aplicaciones de prácticas interactivas, desarrolladas en conjunto por los docentes de la asignatura, que permiten a los alumnos adquirir habilidad en el manejo de los temas abordados de métodos de generación de números pseudoaleatorios, muestras artificiales y pruebas de hipótesis para la verificación de los mismos, y la modelización y simulación de problemas. Estas aplicaciones constituyen simuladores de los temas abordados en las unidades de la asignatura y están orientadas a afianzar la comprensión de los mismos. Se implementan como complemento educativo en las clases tradicionales, y son incorporadas 
luego al CD de la asignatura. Presentan un entorno dinámico que permite el ingreso de los parámetros requeridos y el análisis de los elementos de un modelo y sus interrelaciones.

j) Autoevaluativos: [28] considera que "el diseñar estrategias de evaluación más específicas basadas por ejemplo herramientas de la web 2.0, las cuales permiten llevar a cabo dinámicas de evaluación activas y participativas". En coincidencia con lo expuesto, se elaboraron autoevaluaciones que implementan ejercicios del tipo de múltiple elección y crucigramas, entre otros.

k) Herramientas: descarga de programas de utilidad y para emplear en la realización de los Trabajos Prácticos (Acrobat Reader, Winzip, etc.).

l) Consultas: Formulario interactivo para facilitar las consultas de los estudiantes a los docentes.

m) El CD- ROM conteniendo el EVEA se actualiza en cada ciclo lectivo, con material didáctico teóricopráctico elaborado ad-hoc, guías de laboratorio, guías de trabajos prácticos, ejercicios de simulación, autoevaluaciones, etc.

n) A continuación se describe el módulo incorporado al EVEA de la asignatura Modelos y Simulación, orientado a facilitar un mecanismo para afianzar las prácticas, compuesto por un paquete en Mathematica, un simulador del funcionamiento del paquete y autoevaluativos.

\subsubsection{Funcionalidades del Paquete en Mathematica}

La librería se compone de una serie de procedimientos desarrollados para generar muestras artificiales de variables aleatorias discretas y continuas, empleando métodos especiales. Las Figuras 1.7 y 1.8 muestran el código y la salida obtenida al ejecutar el procedimiento Poisson(), que permite generar una muestra artificial que sigue esta distribución empleando un método especial.

Por otra parte, como lo señala [8], una de las ventajas de "la producción de este tipo de instrumentos es que una vez que se tiene el concepto de lo que se quiere graficar y se ha hecho al menos un prototipo, es más fácil modificar y perfeccionar el instrumento a partir de este primero". Es decir, es posible reutilizar el código que permite generar representaciones gráficas a partir de las funciones proporcionadas por las herramientas o entornos de programación orientados al cálculo matemático como MatLab, Mathematica, Octave. Es así como una función de graficación puede adecuarse para la resolución de otros problemas similares.

\subsubsection{Simuladores del funcionamiento del paquete}

Los simuladores son herramientas informáticas que permiten simular un determinado proceso como si fuera real. Su uso en educación es enfocado al aprendizaje de contenidos y la resolución de problemas.

En este trabajo se los implementó con fines ilustrativos del funcionamiento del paquete construido. Pueden ser concebidos como una extensión de la ayuda disponible. En la Figura 1.9 muestra el funcionamiento de algunos simuladores, que mediante animaciones permiten visualizar la ejecución de algunos métodos.

\subsubsection{Autoevaluaciones}

Las evaluaciones o autoevaluaciones digitales brindan a los estudiantes alternativas para afianzar y/o validar sus conocimientos. La Figura 1.10 ilustra el funcionamiento de una autoevaluación y la visualización de las respuestas correctas 


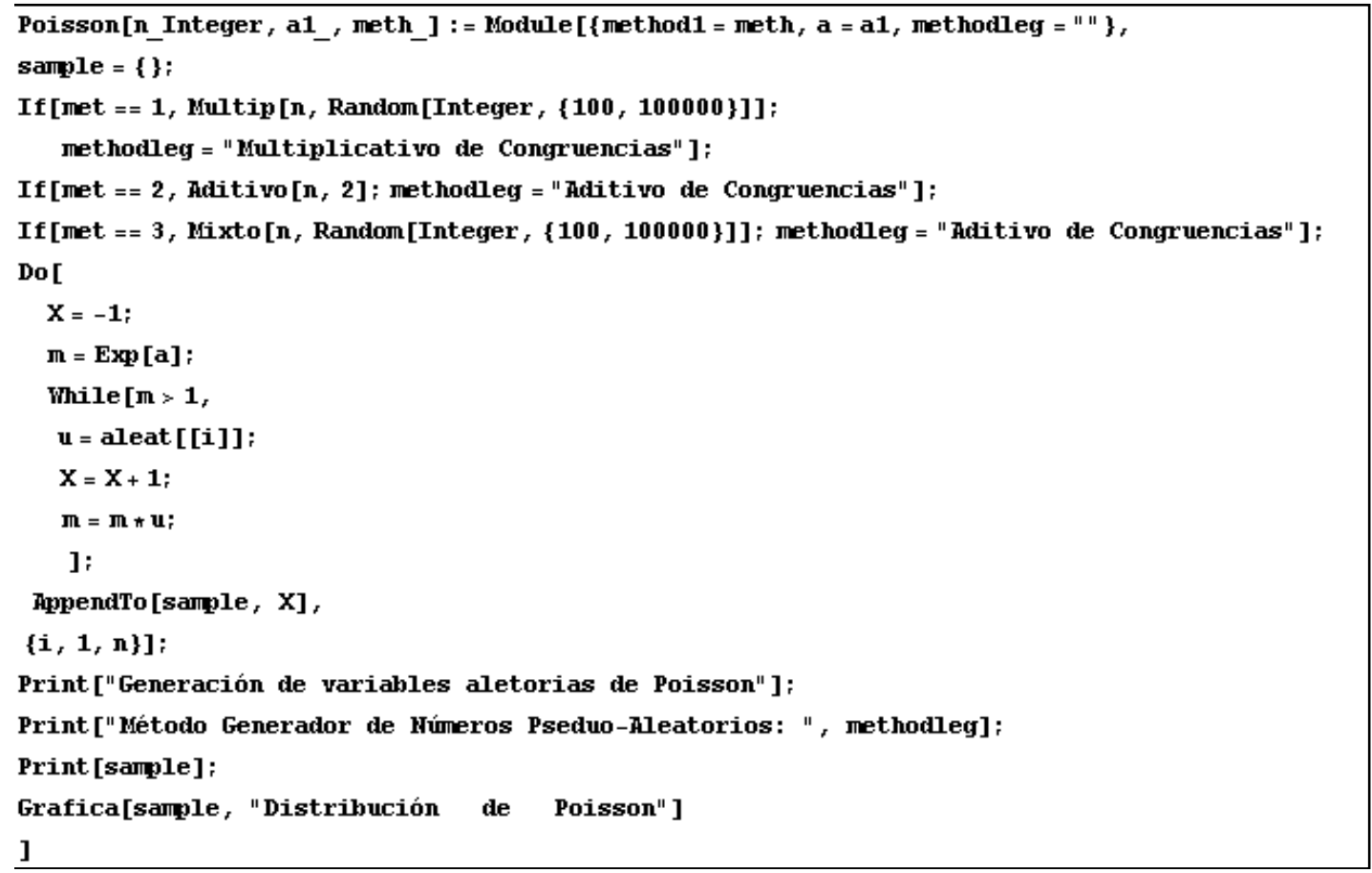

Figura 1.7 Procedimiento Poisson() escrito en Mathematica, que implementa un método especial para generar una muestra artificial que sigue esta distribución

Poisson $[250,0.75,1]$

Generación de variables aletorias de Poisson

$\{1,1,1,2,2,1,0,0,1,1,1,0,2,1,0,2,4,1,1,0,1,1,1,0,0,0,1,1,0,0,2,0,0,0,2$, $0,0,1,1,0,0,1,0,1,0,2,1,1,3,1,0,0,1,2,1,2,1,0,0,0,1,1,0,1,0,0,0,1,0$, $0,0,0,1,0,0,2,0,0,1,1,1,0,1,0,1,1,1,1,0,2,0,1,1,0,0,1,1,0,1,1$, $0,3,0,1,0,0,1,2,1,0,0,0,0,1,0,1,0,1,1,0,0,0,0,2,1,1,0,1,0,0$, $0,0,1,2,0,1,2,1,0,1,0,0,0,1,2,1,1,1,0,3,0,1,1,1,1,0,1,0,1,0$, $3,1,2,0,1,0,1,0,1,0,2,0,1,2,3,0,1,2,0,3,1,0,1,1,1,1,3,0,1,1$, $1,1,0,0,3,2,1,0,0,0,0,1,2,1,1,0,0,1,1,0,0,0,2,1,2,0,0,0,2,0$, $1,1,0,0,1,1,1,1,0,0,0,1,0,0,3,0,1,1,1,0,3,1,0,2,2,1,3,3,4,0\}$

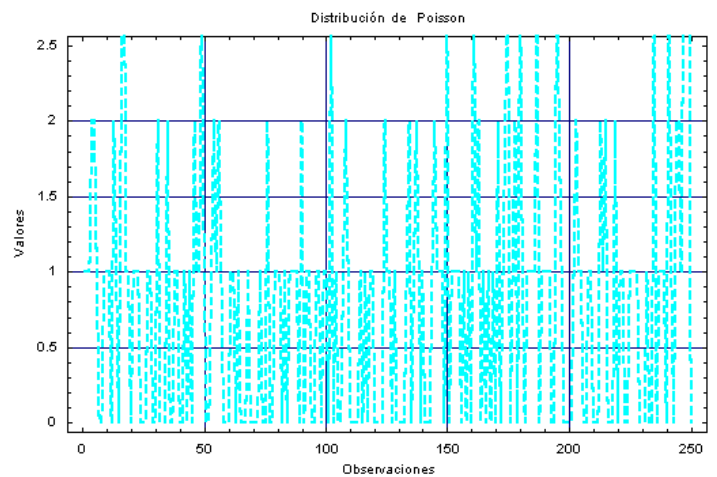

Figura 1.8 Resultados obtenidos el ejecutar el procedimiento Poisson() 


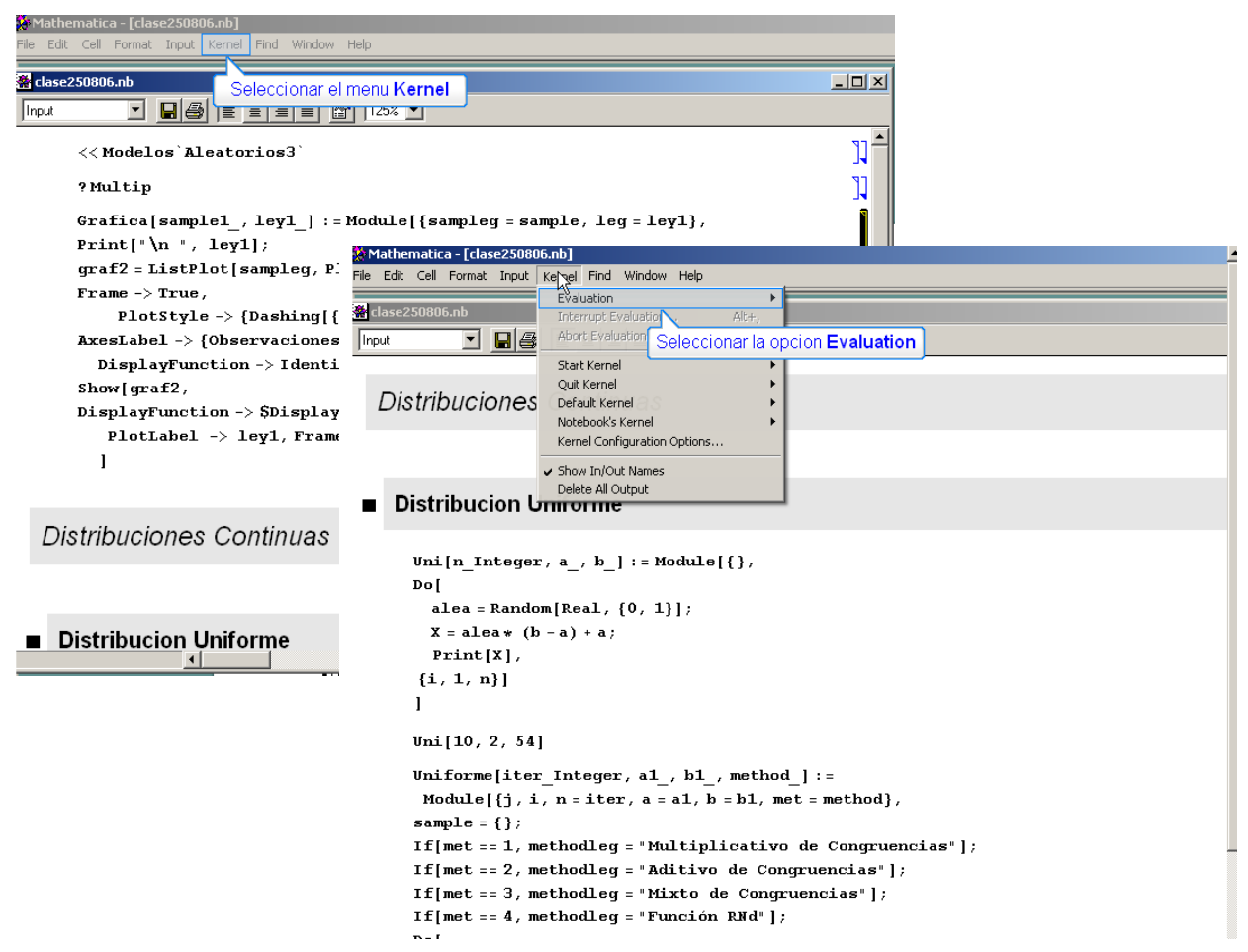

Figura 1.9 Visualización de simuladores del funcionamiento del paquete en Mathematica

Figura 1.10 Visualización de una autoevaluación y sus respuestas correctas

\subsubsection{El empleo del paquete en el aula}

En el EVEA de la asignatura se incluyen los paquetes de Mathematica, los cuales son empleados en las prácticas de laboratorio propuestas por la asignatura. La utilización y manipulación de estos paquetes permite a los estudiantes:

a) Usar las computadoras en la abstracción y el tratamiento de problemas reales, mediante la experimentación con diferentes ejercicios de simulación.

b) Disponer de una herramienta complementaria para afianzar e integrar los contenidos abordados en los ejes temáticos de la asignatura.

c) Repasar conceptos fundamentales de la asignatura.

d) Diseñar y desarrollar experimentos a fin de evaluar el rendimiento de los resultados proporcionados por los diferentes métodos programados en Mathematica [22], y su comportamiento con respecto a otros similares programados en otros lenguajes de programación, como Octave [33].

e) Aplicar el paquete para modelizar y simular abstracciones de simulaciones reales.

Durante las clases Teórico-Prácticas de la asignatura, se proponen a los alumnos la resolución las Guías de Trabajos Prácticos, para lo cual se requiere del alumno el conocimiento previo de los contenidos teóricos, permitiéndole resolverlas en términos de aprendizaje. Mediante el desarrollo de las series de trabajos prácticos, 
se espera lograr que los alumnos efectúen la formalización de los algoritmos correspondientes a los métodos de generación de números pseudoaleatorios, muestras artificiales de variables aleatorias discretas y continuas y modelos de simulación de problemas planteados durante el desarrollo de la asignatura, mediante las técnicas de diagrama de flujo y/o pseudocódigo, con el objeto de su posterior procesamiento mediante computadoras. Con el objeto de lograr la conexión con el campo profesional y disciplinar, se incorporan a las series de trabajos prácticos ejemplos basados en situaciones reales de dominio técnico o académico/científico, para ilustrar a los futuros egresados cómo estos problemas pueden resolverse empleando modelos de simulación. Se busca formar a los alumnos en el desarrollo de aplicaciones tanto de tipo científico como del campo de las organizaciones o empresas, constituyendo éstos dos aspectos diferentes de su formación profesional. Los mismos deben ser capaces de identificar las características básicas de los problemas, y resolverlos empleando la metodología de modelos de simulación utilizando la computadora.

Seguidamente, en las clases de Laboratorio de la asignatura, los alumnos acceden a las aplicaciones del paquete en Mathematica, empleando los simuladores de funcionamiento del mismo descriptos. Estas aplicaciones de prácticas interactivas permiten a los alumnos adquirir habilidad en el manejo de métodos de generación de números pseudoaleatorios, muestras artificiales y pruebas de hipótesis para la verificación de los mismos, y la modelización y simulación de problemas, temas abordados en las unidades de la asignatura, y están orientadas a afianzar la comprensión de los mismos. Presentan un entorno dinámico que permite el ingreso de los parámetros requeridos y el análisis de los elementos de un modelo y sus interrelaciones, lo que posibilita a los alumnos afianzar los conocimientos adquiridos en las clases Teórico-Prácticas a medida que avanzan en la lectura y estudio de los contenidos teóricos, y efectuar auto-evaluaciones del aprendizaje de manera continua.

Las clases de Laboratorio tienen como objetivo el entrenamiento de los alumnos en la programación y el procesamiento mediante computadoras de los ejemplos prácticos expuestos en el desarrollo de las clases Teórico-Prácticas, actividad que es orientada mediante las Guías de ensayos de Laboratorio, cuya resolución debe incluir: a) Planteo del problema; b) Metodología aplicable para resolverlo, c) Descripción de variables y parámetros intervinientes; d) Diagrama de flujo o pseudocódigo correspondiente, e) Prueba de escritorio; f) Codificación en una herramienta o lenguaje de programación; g) Informe en donde se incluyan, al menos, los resultados obtenidos con cinco ejecuciones del programa. Para el desarrollo de estas Guías de ensayo de laboratorio los alumnos pueden elegir el lenguaje Mathematica u otro.

Para evaluar la aceptación del EVEA de la asignatura y del paquete en Mathematica por parte de los alumnos, se aplicó una encuesta anónima en línea a los alumnos que cursaron la asignatura entre los años 2008-2010 (Figura 1.11). Ingresaron al sitio de la encuesta 17 ex alumnos, de los cuales 10 completaron el cuestionario elaborado, y el resto sólo inició este proceso.

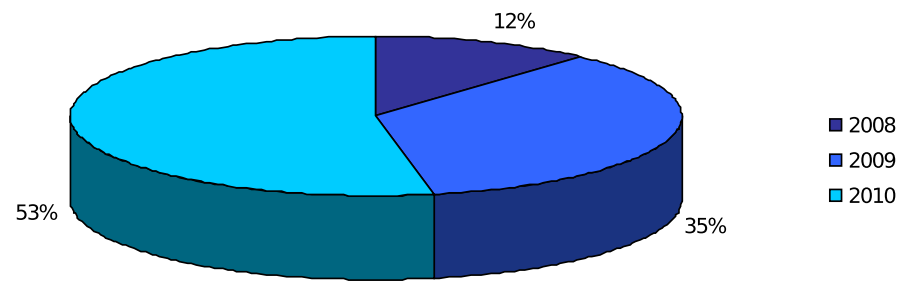

Figura 1.11 Año en que el alumno cursó la asignatura

El 78\% de los alumnos encuestados considera que el EVEA de la asignatura fue fácil de manejar. Consultados acerca de si éste presentó problemas de acceso, el 67\% respondió negativamente, un $11 \%$ respondió que medianamente, y un $22 \%$ contestó positivamente. Entre algunos problemas se mencionan que el EVEA no 
funciona adecuadamente los navegadores diferentes de Internet Explorer y cuando Javascript se encuentra desactivado.

Esta información de realimentación permitirá al equipo docente de la asignatura iniciar estudios para mejorar la accesibilidad en los entornos de enseñanza - aprendizaje.

Por otra parte, se detectó que un mismo grupo de alumnos (30\%) contestó negativamente sobre cuestiones referentes a la evaluación del paquete en Mathematica: si permitió afianzar los temas, si facilitó la comprensión del material de estudio, si al interfaz y la ayuda del paquete resultaron claras y precisas. Esto podría deberse a que en otras asignaturas de la carrera se utilizan otros paquetes científicos como MatLab, y al disponer de un conocimiento previo, los alumnos encuentran dificultoso el estudio de otros paquetes, o prefieren continuar utilizando los lenguajes de programación que ya conocen para desarrollo de los modelos de simulación, en lugar de emplear un tiempo extra en el aprendizaje de un nuevo lenguaje.

La característica de anónima de la encuesta constituyó un obstáculo para realizar el cruce de las respuestas "negativas" de los alumnos, con sus calificaciones y evaluaciones, considerándose este un aspecto negativo del estudio realizado, a mejorar en futuros relevamientos de datos.

Por lo expuesto, desde la asignatura se promoverá el uso de lenguajes y paquetes de programación empleados en otras asignaturas y comúnmente utilizados por los alumnos (trabajo inter-cátedra), para el desarrollo de los productos o modelos de simulación solicitados.

\subsection{Conclusiones}

Se describió un módulo de EVEA de la asignatura Modelos y Simulación de la FACENA (UNNE), orientado a apoyar el proceso de enseñanza-aprendizaje referido a la construcción de muestras artificiales de distribuciones discretas y continuas empleando métodos especiales. El paquete de funciones y otros recursos incorporados como los simuladores y autoevaluaciones constituyen una herramienta apreciable, para lograr la apropiación de los conceptos requeridos como a priori para la construcción de modelos de simulación.

Así mismo, el empleo de los simuladores posibilitará que los estudiantes observen cómo la modificación de un determinado parámetro en un método, se ve reflejada de forma inmediata en los resultados obtenidos. Mediante el uso de os simuladores, el control del proceso de aprendizaje (por descubrimiento) es llevado por el estudiante y no por la computadora, lo cual permite al primero la asimilación de los conceptos a través de la exploración, el descubrimiento y la experimentación.

De este modo, se intenta crear un ámbito de formación continua en temas específicos de la asignatura, mediante la implementación de innovaciones pedagógicas, la elaboración de materiales didácticos en diversos formatos y el tratamiento interdisciplinario de los diversos temas del programa de la asignatura.

Por otra parte, en concordancia con la política institucional de la Universidad y la Facultad de promover el acceso y el desarrollo de cátedras desde la plataforma UNNE-Virtual, se prevé incorporar el recurso didáctico descripto como una herramienta más disponible desde el espacio virtual asignado a la asignatura Modelos y Simulación. 
Agradecimiento: Al Sr. Carlos Primorac por colaborar en el diseño e implementación de la encuesta y el posterior procesamiento de la misma.

\section{Bibliografía}

[1] Barreto, S. E.; Petroff Coloff, N.; López, M. V.; Mariño, S. I. Modelado y simulación de una represa hidroeléctrica. XXIV Encuentro Nacional de Docentes en Investigación Operativa (ENDIO). XXII Escuela de Perfeccionamiento en Investigación Operativa (EPIO). Universidad Tecnológica Nacional. Facultad Regional Buenos Aires. Buenos Aires. Mayo de 2011. 12 pgs. 2011.

[2] Bogado, N.; López, M. V.; Mariño, S. I. Simulando modelos de existencias en la asignatura Modelos y Simulación. Encuentro BTM Web 2010. Septiembre de 2010. 6 pgs. 2010.

[3] Brennan M. Blended Learning and Business Change. Chief Learning Officer Magazine. 2004.

[4] Bravo Ramos. J. L., Sánchez Núñez, J. A., Farjas Abadíam, M. El uso de sistemas de b-learning en la enseñanza universitaria. En: http://www.ice.upm.es/wps/jlbr/Documentacion/Uso_ b-LearComu.pdf Consulta: 04/01/2010.

[5] Coss Bu, R. Simulación. Un enfoque práctico. Ed. LIMUSA. 1991.

[6] Craveri, A. M.; Spengler, M. El uso de TIC en el aprendizaje de la matemática básica universitaria. Universidad Nacional de Rosario. Universidad Tecnológica Nacional. Argentina.

[7] Chelquer, S. J.; Mutis Vadalá, A. Modelado y TICs en la enseñanza de ciencias y Matemática. VIII Congreso internacional sobre investigación en la didáctica de las ciencias 2009.En: http: //ensciencias.uab.es.2009.

[8] Díaz Navarro, P. Aspectos Técnicos y Metodológicos para el Diseño de Instrumentos Educativos Multimediales de Cálculo Diferencial e Integral. Revista virtual Matemática, Educación e Internet. En: http://www.cidse.itcr.ac.cr/revistamate/MundoMatematicas/ aspectostecnicos.htm Consulta: 04/01/2010.

[9] García Barneto, A.; Gil Martín, M. Entornos constructivistas de aprendizaje basados en simulaciones informáticas. Revista Electrónica de Enseñanza de las Ciencias Vol. 5 Nº 2. 2006

[10] Giacosa, N.; Maidana, J.; Beck, S.; Von der Heyde, W. Análisis de simulaciones de acceso libre para el estudio del Ciclotrón. Congreso BTM 2007. Punta del Este. Uruguay. 2007.

[11] Fainholc, B. Optimizando las posibilidades de las TICs en Educación. Edutec. Revista Electrónica de Tecnología Educativa, $\mathrm{N}^{o} 22$.

[12] Ferreira Szpiniak, A.; Sanz, C. Hacia un modelo de evaluación de entornos virtuales de enseñanza y aprendizaje. La importancia de la usabilidad. Anales del XIII Congreso Argentino de Ciencias de la Computación. CACIC 2007. En: http://edutec.rediris.es/Revelec2/revelec22/beatriz. pdf.2007.

[13] Gil Chaveznava, P. Diseño curricular y los diversos modelos educativos. Universidad Autónoma Metropolitana. Unidad Iztapalapa. México D. F. En: http://cbi.izt.uam.mx/content/eventos_ divisionales/Seminarios/Seminario_Diseno_Curricular/Modelo_educativo_y_Plan_ estudio.pdf 2007.

[14] González Mariño, J. C. B-Learning utilizando software libre, una alternativa viable en Educación Superior Revista: Revista Complutense de Educación, 2006; 17 (1): 21-133. En http://revistas . ucm.es/edu/11302496/articulos/RCED0606120121A.pdf. 2006.

[15] Gordon, G. Simulación de sistemas. Ed. Diana. 1981.

[16] López, M. V., Mariño, S. I. Desarrollo y evaluación de un modelo b-learning de enseñanza-aprendizaje en una asignatura de la carrera de Sistemas. Edutec 2007. Universidad Tecnológica Nacional. Edutec: 
Asociación para el desarrollo de la tecnología educativa y de las nuevas tecnologías aplicadas a la educación. Publicado en Actas. ISBN: 978-950-42-0088-8. 9 pgs. Buenos Aires. Argentina. 2007.

[17] López, M. V.; Mariño, S. I. Simuladores para afianzar conceptos de teoría de colas. Un caso de estudio. XV Congreso Argentino de Ciencias de la Computación (CACIC 2009). Universidad Nacional de Jujuy. Octubre de 2009. ISBN: 978-897-24068-4-1. 10 pgs. 2009.

[18] López, M. V., Mariño, S. I. Relevamiento de datos de los estudiantes de la asignatura 'Modelos y Simulación' de la FACENA para definir el perfil en referencia al acceso a las TICs. VII Encuentro Regional de Docentes de Matemática. Facultad de Ciencias Exactas y Naturales y Agrimensura. Universidad Nacional del Nordeste. Corrientes. Argentina. 2007.

[19] López, M. V., Mariño, S. I. Propuesta de innovación a la hora de evaluar en la asignatura Modelos y Simulación. Inédito. 2010.

[20] Mariño, S. I., López, M. V. Aplicación del modelo b-learning en la asignatura 'Modelos y Simulación' de las carreras de Sistemas de la FACENA- UNNE. Edutec: Revista Electrónica de Tecnología Educativa. España. ISSN: 1135-9250. N $\Sigma 23.14$ pgs. Disponible en: http://edutec.rediris.es/Revelec2/ revelec23/revelec23.html.2007.

[21] Mariño, S. I., López, M. V. La simulación de sistemas en un entorno integrado de b-learning. Anales del Encuentro Internacional BTM 2007 Educación, formación y nuevas tecnologías. Utemvirtual. Universidad Tecnológica Metropolitana. Punta del Este, Uruguay. 2007.

[22] Mariño, S. I. y López, M. V. Generadores de números aleatorios. Ed. Moglia. ISBN 978-987-05-5025-0. 2008.

[23] Mariño, S. I.; López. M. V. Un proyecto de docencia, extensión e investigación en la asignatura Modelos y Simulación. Anales del X Workshop de Investigadores en Ciencias de la Computación. X WICC. ISBN 978-950-863-863-101-5. 2008.

[24] Mariño, S. I. y López, M. V. Propuesta metodológica para la construcción de software educativo en la asignatura Modelos y Simulación. Anales de XXII ENDIO y XX EPIO. 2009.

[25] Mariño, S. I.; López. M. V. Avances del proyecto de docencia, extensión e investigación en la asignatura "Modelos y Simulación””. Anales WICC 2010 - XII Workshop de Investigadores en Ciencias de la Computación. XII WICC. 682-686. 2010.

[26] Mantiega Gonzalez, M., T. Perez De Vargas, A Estadística Aplicada. Una visión instrumental. Ediciones Dias de Santo. 2009.

[27] Martins A., Fracchia C. C., Allan C., Parra, S. Simulación y métodos numéricos en ciencias de la computación: uso de TICs. Anales WICC 2010 - XII Workshop de Investigadores en Ciencias de la Computación. XII WICC. 739-744. 2010.

[28] Mas, X., Gros, B., García, I. La evolución del modelo educativo de La UOC: un enfoque orientado a la Adquisición de las competencias del Siglo XXI. Anales del X Virtual Educa. 2009. http://www . virtualeduca.info/.2009.

[29] Mortis Lozoya, S. V. Efectividad de los Objetos de Aprendizaje en un Curso Modalidad mixta. Anales del X Virtual Educa. http://www.virtualeduca.info/ 2009.

[30] Naylor, T., Balintfy, J. Burdick, D., Chu, K. Técnicas de simulación en computadoras. Ed. Limusa. 1975.

[31] Pace, G. J., Mariño, S. I., López, M. V. Material Didáctico de la cátedra Modelos y Simulación. FACENA. UNNE. Inédito. Corrientes. Argentina. 2010. Inédito.

[32] Pardo, L. y Valdés, T. Simulación - Aplicaciones Prácticas en la empresa. Ed. Díaz de Santos S.A. 1987.

[33] Pérez, C.; Mariño, S. I.; López, M. V. Desarrollo de generadores de números pseudoaleaorios en Octave. Anales del IV Congreso de Tecnología en Educación y Educación en Tecnología '09, 6774pp.Universidad Nacional de la Plata. 2009.

[34] Primorac, C.; López, M. V.; Mariño, S. I. Construcción de una librería de números pseudoaleatorios y muestras artificiales con Matlab. Revista "Investigación Operativa". Escuela de Perfeccionamiento en 
Investigación Operativa “Isidoro Marín”. Universidad Tecnológica Nacional. Facultad Regional Buenos Aires. Buenos Aires. Argentina. ISSN: 0329-7322. En prensa. A publicarse en el N $\Sigma$ 32. Sección "Enseñanza". 17 pgs. 2010.

[35] Primorac, C.; Mariño, S. I.; López, M. V. Programación en Octave de una librería de métodos especiales para generar muestras artificiales de variables aleatorias discretas. II Encuentro Regional Argentino Brasilero de Investigación Operativa (ERABIO), XXIII Encuentro Nacional de Docentes en Investigación Operativa (ENDIO) y XXI Jornadas de la Escuela de Perfeccionamiento en Investigación Operativa (EPIO). Septiembre de 2010. 14 pgs. 2010.

[36] Primorac, C.; Mariño, S. I.; López, M. V. Simuladores para afianzar conceptos de modelos de existencias. Un caso de estudio. V Congreso de Tecnología en Educación y Educación en Tecnología (TE\&ET 2010). Universidad Nacional de la Patagonia Austral. Mayo de 2010. ISBN:978-987-1242-42-9. 10 pgs. 2010.

[37] Yebra, M.; Chuvieco, E. Modelos de Simulación de Reflectividad en ecología: potencialidades y problemas. Ecosistemas, 17(3):23-38. Septiembre 2008. http://www.revistaecosistemas.net/ articulo. asp? Id=568.2008. 